\title{
Advanced Spacecraft Designs In Support Of Human Missions To Earth's Neighborhood
}

\author{
David Fletcher
}

Advanced Design Office, NASA Johnson Space Center, Houston, TX 77058

\begin{abstract}
NASA's strategic planning for technology investment draws on engineering studies of potential future missions. A number of hypothetical mission architectures have been studied. A recent study completed by The NASA/JSC Advanced Design Team addresses one such possible architecture strategy for missions to the moon. This conceptual study presents an overview of each of the spacecraft elements that would enable such missions. These elements include an orbiting lunar outpost at lunar L1 called the Gateway, a lunar transfer vehicle (LTV) which ferries a crew of four from the ISS to the Gateway, a lunar lander which ferries the crew from the Gateway to the lunar surface, and a one-way lunar habitat lander capable of supporting the crew for 30 days. Other supporting elements of this architecture discussed below include the LTV kickstage, a solar-electric propulsion (SEP) stage, and a logistics lander capable of re-supplying the 30-day habitat lander and bringing other payloads totaling $10.3 \mathrm{mt}$ in support of surface mission activities. Launch vehicle infrastructure to low-earth orbit includes the Space Shuttle, which brings up the LTV and crew, and the Delta-IV Heavy expendable launch vehicle which launches the landers, kickstage, and SEP.
\end{abstract}

\section{INTRODUCTION}

The Gateway Architecture is a feasibility study which provides one possible means of returning humans to the moon within the next ten years while providing development of core capabilities that will enable human missions to Mars. Such core capabilities include the development of advanced systems and technologies that can be developed and tested in a near-earth operational environment. Such an environment will provide operational experience for autonomous deep space operations, planetary surface operations, and a Mars analog operations base at the lunar south pole.

A significant return from scientific activities may result from investigations on the lunar surface. These include a clearer understanding of the impact history of comets in near-earth space, better knowledge about the composition of the lunar mantle, past and present solar activity, lunar ice at the poles, and the history of volatiles in the solar system. Commercial potential includes the extraction of oxygen, water and metals from the lunar soil, and materials processing.

Several important assumptions are made at the outset to enable the development of the mission architecture. These include deferring the development of high-capacity launch systems by utilizing existing launch vehicle systems, and utilizing lunar libration point number one and the International Space Station (ISS) as transfer nodes between the two planetary surfaces. In addition, no long-term commitment regarding extensive lunar surface infrastructure is made while initial transportation capabilities are established allowing for the future expansion of science and commercialization activities. Finally, a crew of four can be transported to and from the moon for expeditionary missions or for extended stay missions and returned to earth. Any cargo to the lunar surface is transported separately from the crew and is pre-deployed on the lunar surface before the crew arrives.

The Gateway Architecture is composed of a suite of elements which make it possible to send and return humans from the moon. These elements include a lunar depot called the Gateway which is located at L1, the Lunar Transfer Vehicle (LTV) which ferries the crew from the International Space Station to the Gateway, a high-energy injection stage which provides an initial boost for the LTV, the L1 Lunar Habitat Lander which supports the crew for 30-days at the lunar south pole, the L1 Lunar Lander which performs three-day expeditionary missions to any point on the lunar surface or 30-day extended missions at the lunar south pole, and high-efficiency solar electric propulsion transfer vehicles which spiral the Gateway and landers to the L1 staging area. Other supporting elements of the architecture include the Space Shuttle which launches crew to the ISS and the Gateway to low 
earth orbit, the ISS which houses the LTV and serves as the nominal terminal for returning lunar astronauts, the Delta-IV expendible launch vehicle which brings the LTV and landers to low earth orbit, the Global positioning system for navigation, and an Lunar positioning system to aid in lunar navigation and communication with earth. The figure below depicts how the Gateway Architecture elements are deployed to perform the mission.

\section{THE GATEWAY}

The Gateway is an outpost located at L1 whose purpose is to accommodate a crew of four in support of lunar surface missions, telescope outfitting missions, and possibly Mars missions. Lunar libration point $1(\mathrm{~L} 1)$ was selected since it provides global lunar surface access from the Gateway, no orbital debris at unstable points, continuous sunlight (>99.9\%), full-sky viewing, continuous direct communication with Earth, low station-keeping requirements $(\sim 10 \mathrm{~m} / \mathrm{sec} / \mathrm{yr})$, deep space environment within Gateway, and injection opportunities back to the international space station every 10-11 days.

The Gateway is designed to support a crew of four over a period of fifteen years for up to 42-days continuously for each mission. This facility is capable of supporting four docked vehicles in a dormant mode, has external payload manipulation capability, and can support two-person extra-vehicular activities (EVA's) for telescope construction. The Gateway provides radiation protection against solar particle events and provides an internal pressure of $7.03 \times 10^{4} \mathrm{~Pa}(10.2 \mathrm{psia})$ mixed oxygen/nitrogen atmosphere which minimizes EVA pre-breathe and reduces structural requirements.

Once it reaches low-earth orbit, the Gateway is propelled by a high-efficiency, low-thrust solar-electric propulsion (SEP) system with 2,500 sec Isp Hall thrusters which transfers the facility in a continuous spiral towards lunar $\mathrm{L} 1$ over a period of $4 \frac{1}{2}$ months. The vehicle is launched from the earth with existing launch vehicle technology including such vehicles as the Space Shuttle and Delta-IV-Heavy launch vehicle systems. The Gateway is constrained to a maximum of two launches to low-earth orbitone of which will be a Shuttle outfitting mission to deploy external or internal equipment and to perform vehicle checkout prior to its transfer to L1. Following the docking of the Gateway with its SEP transfer stage the docked pair is released from the Shuttle whereupon the low-thrust, high-efficiency engine commences firing continuously for 143 days to transfer the unmanned Gateway to its final destination at lunar L1. Shortly after arriving at L1 the Gateway awaits the arrival of the first L1 Lunar Lander which will ferry a crew of four to the lunar surface and back again. Once it arrives at the Gateway the L1 Lunar Lander docks on an axial docking node and is then transferred to an empty berthing port by a robotic arm mechanism similar to the Shuttle remote manipulator system (RMS) or International Space Station remote manipulator system (ISSRMS). The Lunar Transfer Vehicle then brings a crew of four to the Gateway either to transfer to the L1 Lunar Lander for lunar surface missions, or for construction and deployment of Gossamer telescopes to earth-solar L2.

Current plans call for leveraging the development of the Gateway element concept based on the development work that has already been completed by the JSC Transhab design team. (The Transhab project was a JSC-sponsored technology development program at JSC which developed crewed inflatable habit technologies to support the ISS habitat module and for eventual Mars missions.)

\section{THE L1 LUNAR LANDER}

\section{Element Description}

The L1 Lunar Lander (L1LL) is the crew transportation element in the Gateway Architecture which ferries the crew from the Gateway to the Lunar surface and back again. The lander is capable of supporting a crew of four for a total of nine days-three of which are spent on the lunar surface. The lander is comprised of two stages-an ascent and a descent stage. The descent stage is composed of landing gear, main propulsion system descent tanks, descent reaction control system (RCS), and support structure while the ascent stage hosts the crew module, avionics, the environmental control and life support system (ECLSS), ascent propulsion tanks, ascent RCS, and main propulsion system. In order to minimize the payload mass to the Gateway, the descent stage is left behind on the lunar 
surface. In addition to the crew, the ascent stage is capable of delivering $50 \mathrm{~kg}$ of Lunar samples to the Gateway for transfer back to Earth for scientific analysis.

The L1 Lunar Lander is designed to fulfill two types of missions. The first of these missions is of the expeditionary-type, where the lander is capable of sustaining a crew of four for three days at any location on the Lunar surface. In this mode, the crew uses the lander as its primary base and habitation base camp for short duration missions. The second mission for the L1 Lunar Lander is to ferry the crew to and from the L1 Lunar Habitat Lander located at the Lunar south pole. In this mission, the crew will live in a 30-day habitat module for extended Lunar missions while the L1 Lunar Lander awaits crewed ascent on stand-by in survival power mode.

Housed on the descent stage is an unpressurized rover capable of transferring the crew to and from the L1 Lunar Habitat Lander (L1LHL) and which is also used as a mobility aid for crew traverses of the Lunar surface during extra-vehicular activities (EVAs). In addition to the rover, the descent stage also houses a pallet containing science payloads for use during expeditionary surface missions. Alternately, this payload pallet could be used to resupply the L1 Lunar Habitat Lander.

\section{L1 Lunar Lander Design Objectives, Constraints \& Requirements}

\section{Objectives}

- Global lunar access from the Gateway

- TRL of 6 by 2005 for all vehicle system technologies

Constraints

- Use existing launch vehicle technology - i.e., Delta-IV-Heavy expendable launch vehicle with a proposed payload capability of 35.4 metric tons to the ISS

\section{Requirements}

- Crew of four (4)

- Mission duration-nine (9) days

- Reusability-non-reusable

- Stageability - two-stage vehicle which stages on the lunar surface

- Total propulsive delta- $\mathrm{V}-5,562 \mathrm{~m} / \mathrm{sec}$

- The L1 Lunar Lander shall carry a $240 \mathrm{~kg}$ unpressurized rover and a minimum of $430 \mathrm{~kg}$ of science equipment on the descent stage

- Ascent stage shall return the crew and a minimum of $50 \mathrm{~kg}$ of lunar samples to the Gateway

- The L1 Lunar Lander shall be capable of precision landing and hazard avoidance with manual

- The tidleunar Lander shall be delivered to the Gateway via a solar electric propulsion (SEP)

- Tthgerew cabin shall be depressurized for surface EVA's

- The L1 Lunar Lander shall be capable of providing up to four cabin pressurizations

- The main propulsion system shall employ a 2:1 oxygen to fuel ratio-throttleable LOX/Methane

- Ithginel Lunar Lander shall have abort-to-surface (engine-out), abort-to-L1 (Gateway) capability

- Rendezvous and docking maneuvers shall be automated with manual override capability

- The L1 Lunar Lander shall maintain a cabin pressure of 10.2 psia

- The L1 Lunar Lander shall be launched within a $6 \mathrm{~m}$ diameter by $18 \mathrm{~m}$ height launch payload shroud

\section{Vehicle Configuration}

In order to minimize the crew access height and to eliminate the issues associated with shifting center of gravity, a horizontal lander concept that would be launched on-end in the payload shroud was 
considered. The symmetrical design simplifies the center-of-gravity thrust profile while tank configurations are either spherical or cylindrical to minimize mass. The on-end launch configuration allowed the lander length to grow beyond the 6 meter launch shroud diameter so that upon landing the crew egress height could be minimized to within 2.5 meters of the Lunar surface.

Furthermore, radiators for thermal control could be mounted on top of the crew cabin instead of deploying them thus reducing vehicle complexity. Due to its I-beam structure (see discussion below on structures) a platform for crew dust-off was provided so that upon L1LL ingress, Lunar dust would not be tracked into the L1LL. As in the vertical configurations, the ascent stage with crew cabin and main propulsion system separates from the descent stage to leave behind landing gear, descent tanks, and descent structure.

\title{
Vehicle Mass
}

The initial mass of the L1 Lunar Lander at the Gateway is $29,655 \mathrm{~kg}--335 \mathrm{~kg}$ below its original target weight of $30,000 \mathrm{~kg}$ and $5,735 \mathrm{~kg}$ below its maximum-allowed launch weight of $35,400 \mathrm{~kg}$ for a total growth margin potential of $16.2 \%$ While by no means conservative, the potential growth margin percentage is considered satisfactory given that the mass targets for the spacecraft (especially the ascent stage) were so tight.

\section{L1 LUNAR HABITAT LANDER}

\section{Element Description}

The L1 Lunar Habitat Lander (L1LHL) is a surface habitation/science laboratory which can support a crew of four at the lunar south pole for 30 days. The spacecraft is cylindrically-shaped, lands vertically, and is supported by a four-legged main landing gear. The lander is propelled by four liquid oxygen-methane pressure-fed engines, and is powered by fixed and deployable gallium arsenide solar arrays which generate $4.058 \mathrm{~kW}$ peak and $2.42 \mathrm{~kW}$ nominal electrical power for all onboard electrical systems. Configuration of the lander is a vertical cylinder which is divided into three decks. The outer surface of the vehicle serves as the integrated payload launch shroud with a coned portion at the top (which protects the solar arrays and radiators during launch) which is jettisoned during launch to take full advantage of the payload mass and packaging capabilities of the expendible launch vehicle.

The lower deck houses two, two-person airlocks for EVA crew egress/ingress and which can also be used as a radiation shelter to protect the crew during solar events. It also provides an unpressurized porch where the crew may dust off prior to vehicle ingress. Access to and from the surface is provided by deployable stairs. The second deck houses mechanical and avionics systems as well as the science laboratory facilities for processing of Lunar samples, while the third deck houses the crew quarters, galley, and wardroom. Pressurized volume totals $240 \mathrm{~m}^{\wedge} 3$ or $60 \mathrm{~m}^{\wedge} 3$ per crew member. The outside diameter is 6.5 meters while the internal diameter is 6.0 meters.

\section{L1 Lunar Habitat Lander Design Constraints, Objectives \& Requirements}

\author{
Objectives
}

- Crew safety

- Identify enabling system technologies

- System growth margin of 30 percent

- Minimize complexity, cost

- Maximize reliability, maintainability 
- Use existing launch vehicle technology - i.e., Delta-IV-Heavy expendable launch vehicle with a proposed payload capability of 35.4 metric tons to the ISS

- Employ high-efficiency SEP stage to transfer the lander to a circular orbit of $100 \mathrm{~km}$ in low lunar orbit

- Two launch maximum for lander and SEP_-both aboard Delta-IV-H ELV's

- System growth margin of 30 percent

- TRL of 6 by 2005 for all vehicle system technologies

$\bullet$

- Crew of 4

\section{Requirements}

- 30-day surface stay with resupply capability

- 5-year design lifetime

- Landing site at the Lunar south pole

- Total delta $\mathrm{V}=1910 \mathrm{~m} / \mathrm{sec}$ for descent propulsion

- $2: 1$ throttleable LOX/Methane main propulsion system

- LOX/Methane RCS propulsion system

- Single main propulsion system engine-out capability

- Precision landing and hazard avoidance

- 10.2 psia mixed cabin atmosphere $\left(\mathrm{O}_{2} / \mathrm{N}_{2}\right)$

- Science support capability

- Provide crew accommodations and lab support to surface mission

- Provide crew airlocks for crew EVAs

- Provide radiation protection

- Autonomous landing

- Autonomous system startup following the landing

- Provide support to unpressurized rover

- Provide survival power to dormant L1 Lunar Lander

- Sufficient water should be carried aboard the vehicle for the life support system/crew/lab req.

\section{Vehicle Mass}

The initial mass of the L1 Lunar Habitat Lander after separation from the SEP in low Lunar orbit is $27,174 \mathrm{~kg}$ or $8,226 \mathrm{~kg}$ below its maximum allowed launch weight of $35,400 \mathrm{~kg}$ for a total growth margin potential of 23 percent. The final mass statement displayed below demonstrates that the mass of the systems can indeed be accommodated aboard the vehicle while consuming only 7 percent of the initial 30 percent mass growth margin.

\section{LUNAR TRANSFER VEHICLE}

\section{Element Description}

The Lunar Transfer Vehicle (LTV) is the crew transportation element in the Gateway Architecture which ferries the crew from the International Space Station (ISS) to the Gateway located at lunar L1. The LTV is capable of sustaining a crew of four for up to 22 days - nine of which are spent in transit between the ISS and the Gateway. (The additional days allow for the LTV to perform proximity operations for the trans-lunar injection (TLI) kickstage, Gateway, and ISS. It also allows the crew to loiter at the Gateway while waiting for a return opportunity to the ISS in the case where the LTV is unable to dock with the Gateway.) The LTV is composed of two distinct parts. The forward LTV contains the pressurized crew cabin with docking hatch, life support system, power system, avionics, 
crew accommodations (including food and medical supplies), forward reaction control system (RCS), forward aero-shell, suit stowage, and radiation protection system. The aft half of the LTV(called the Logi-pac-short for logistics pack) houses crew consumables (i.e water, breathing gasses), the thermal control system, the LTV main propulsion system return (TEI) stage, contingency parachute landing system, and the aft aero-shell. Following launch, the LTV remains at the ISS when not in use so that it can be reused on subsequent missions in order avoid recurring launch costs, while the logipac is removed following the mission and returned aboard the Shuttle for refurbishment and reflight.

The LTV is designed to satisfy two distinct mission scenarios. The first of these is the nominal mission in which the LTV returns to the ISS following a single-pass aero-capture maneuver. Upon its return to low earth orbit, the LTV returns the crew to the ISS where they transfer to the Shuttle for return to earth. In the interim between missions, the LTV receives resources from the ISS (primarily power to maintain the operation of the thermal control system for minimal systems operation), and EVA support to change-out Thermal Protection System (TPS) ablator panels on the forward LTV. The second mission of the LTV is a one-time return of the LTV and its crew to the surface of the earth in the event of a contingency such as a system failure or crew injury. Once it is determined that a contingency earth return is necessary the LTV performs a direct earth aero-entry maneuver to de-orbit the LTV and its crew through the upper atmosphere with the logi-pac attached to aid with vehicle braking and onboard reaction control system (RCS) for vehicle stabilization. At the end of the entry interface pyro bolts separate the logi-pac from the forward LTV leaving behind a parachute pack attached to the forward LTV via a contingency separation plane. Following logipac separation a drogue chute deploys from the parachute pack to slow the vehicle to sub-sonic speeds. Finally, three ring sail parachutes deploy, and aided by landing attenuation devices onboard the vehicle, gently lowers the LTV and the crew to the ground. The primary objective of this latter mission scenario is to preserve the lives of the crew. Preservation of the LTV for re-flight is not an issue. In addition, the LTV is capable of satisfying a number of contingency abort scenarios throughout its nominal mission-the exact nature of each will be addressed in future work.

Three earth launches are required to bring the LTV and the TLI kickstage to the ISS. The forward section of the LTV is launched aboard a shuttle and is docked at the ISS. A second shuttle is launched to ferry the crew and the Logi-pac to the ISS where the two halves of the LTV are mated. The crew then transfers into the LTV, undocks with the ISS and performs a rendezvous and docking maneuver with the TLI kickstage which was launched to the ISS aboard a Delta-IV Heavy expendable launch vehicle following successful linkup of the LTV and the successful transfer of the crew to the ISS. The kickstage, with its LTV payload, fires and sends the crew to lunar L1 where the Gateway with its L1 Lunar Lander awaits. Following the return of the L1 Lunar Lander to the Gateway, the crew transfers back to the LTV for the return trip to the ISS. Once it has arrived at the earth the LTV uses its aerobrake to slow itself and brakes into the proper orbit for return to the ISS. In the event that the LTV cannot dock with the Gateway an additional 10 days of consumables are carried on board the vehicle in order to allow the LTV at Ll to come in to proper alignment with the return trajectory to the ISS. In the event that the LTV cannot return to the ISS or in the case of crew injury requiring the immediate return to earth, the LTV is capable of performing a one-time direct earth entry to facilitate a crew rescue. In the contingency scenario the vehicle enters the earth's atmosphere, jettisons the Logi-pac, and then deploys a parachute which lowers the vehicle to the ground. In the event that the LTV is unable to dock with the ISS following the completion of its otherwise nominal mission, the crew may perform a contingency EVA to evacuate the LTV and enter the ISS through a station airlock. 


\section{Lunar Transfer Vehicle Design Objectives, Requirements And Constraints}

The following are the final top-level objectives, requirements and constraints for the Lunar Transfer Vehicle:

- Crew safety

$$
\text { Objectives }
$$

- Transfer Crew from ISS to Gateway and back to ISS

- Use existing launch vehicle technology

- Minimize complexity and cost

- Maximize reliability

- Maximize maintainability

- Maximize commonality with existing architecture

- Design for optimum crew use and operations

- Crew of 4

\section{Requirements}

Rationale: Surface mission operations scenario calls for buddy pairs

- Five year vehicle lifetime

Rationale: Need enough re-usability to justify cost and complete mission objectives

- Nominally 2 missions a year Rationale: Maximize re-usability

- LTV is berthed or docked at ISS for up to six months between uses Rationale: The LTV will require basic keep-alive power for its dormant mode

- Total delta-V of 2252 m/sec for return from Gateway, Earth circ @ 51.6 deg and docking w/ Dale: Based upon orbital mechanics requirements of Gateway to ISS

- LTV is capable of crewed rendezvous/docking with kick stage. No EVA required for Botiknge: Levying an EVA requirement upon LTV causes large mass penalty

- LTV is reusable with replacement logistical and fuel module Rationale: Satisfies requirement 1.1 by replacing spent fuel

- Rendezvous and docking capabilities with both ISS and Gateway Rationale: These are the two endpoints of LTV missions

- Contingency EVA capability at ISS Rationale: In case entry into ISS by the regular means is hampered

- Crew must have adequate Radiation Protection during all phases of mission Rationale: Human Space Flight Requirement

- Cabin pressure of 15.3 psi (ISS- overpressure compatible), nominal cabin pressure of 10.2 psi (transit, Gateway, contingency EVA)

Rationale: The 15.3 is to be compatible at ISS, while the 10.2 is no minimize pre-breathe before lunar missions

- 5-g peak acceleration load during aerocapture Rationale: Human Space Flight Requirement

\section{Constraints}

- LTV and kick stage are launched on separate Delta-IV H expendable launch vehicles

- 3-launch maximum combination of shuttle and Delta-IV H for the $1^{\text {st }}$ mission

- 2-launch maximum combination of shuttle and Delta-IV H for subsequent missions

- TRL 6 by 2005 for all systems technology

- $30 \%$ mass growth margin 


\section{L1 LUNAR LOGISTICS LANDER CONCEPT}

\section{Element Description}

The purpose of the L1 Lunar Logistics Lander concept is to provide logistical resupply to the L1 Lunar Habitat Lander at the lunar south pole, and to provide a means of transporting large payloads to the lunar surface in support of surface mission activities. Built upon the basic design of the crewed L1 Lunar Lander, the L1 Lunar Logistics Lander is capable of landing $10.3 \mathrm{mt}$ on the lunar surface with a total package volume of $37 \mathrm{~m}^{3}$. The lander has a mission lifetime of 5 months (including its SEP transfer time), and is a single-use spacecraft. The vehicle has a descent stage with a payload mounted on top that replaces the L1 Lunar Lander ascent stage and crew cabin. The total mass of the vehicle is $29.7 \mathrm{mt}$, is launched to low-earth orbit by a Delta-IV-Heavy expendible launch vehicle, and is transferred to low lunar orbit (100 km circular) by a solar-electric propulsion stage which spirals the L1 Lunar Logistics Lander to the moon in 143 days. Once it arrives in lunar orbit, the lander separates from its SEP stage and performs a propulsive descent and landing at the lunar south pole where it is unloaded once the crew arrives.

The author wishes to emphasize that this concept is approximate in its mass and payload capability and that this design did not receive the full attention of the JSC Advanced Design Team. This concept is however included to show how the crewed Ll Lunar Lander design could be leveraged for logistical resupply of the L1 Lunar Habitat Lander and that the team realizes the need for this type of vehicle in order to enable reusability of the lunar habitat surface infrastructure. It is also included as an enabling element of the Gateway Architecture.

\section{CONCLUSIONS}

The Gateway Architecture has been presented as one possible means of building and testing an infrastructure that could allow humans to venture beyond low-earth orbit within the next ten years. It is the conclusion of the author and the JSC Advanced Design team that while this is one approach for returning humans to the moon, it is not the only one, nor is it necessarily the best approach for doing so.

During the study the JSC Advanced Design Team uncovered several concerns about the architecture that would need to be satisfactorily addressed in order enable the envisioned missions. The LTV will require the use of significant ISS resources as a result of having up to two LTV's parked at the ISS at the same time - one to support crew transfer to the Gateway for lunar surface missions, and another for crew transfer to the Gateway in support of telescope mission. Other LTV concerns are that it requires a large number of launches to bring up new logi-packs and kickstages for each lunar and telescope missions. This will adversely impact the Shuttle launch manifest which will already be strained trying to keep up with ISS maintenance and resupply. The LTV also introduces complexity and risk into the mission in that a rendezvous and docking maneuver must be successfully completed with the kickstage in order to proceed with crew transfer to the Gateway. Also, the LTV TPS is currently a single-use ablative system necessitated by the high temperatures the spacecraft encounters as it performs aerocapture/aeroentry maneuvers. This will require EVAs by the ISS crew to replace the TPS panels followed by inspections to recertify the vehicle for flight. The team also recognizes that the Gateway will require logistical support to resupply critical items such as food and crew consumables, and to bring replacement parts and spares to maintain existing equipment. Furthermore, the crew must become familiar with a plethora of spacecraft in order to safely and successfully execute the prescribed missions. These include the Shuttle, ISS, CRV, LTV, kickstage, L1 Lunar Lander, L1 Habitat Lander, the L1 Logistics Lander, and the Gateway resupply module.

This architecture however serves as a reference point for future studies and has given the JSC Advanced Design Team valuable experience in the system engineering process, conceptual design of architecture elements, concurrent engineering, team building, and automated engineering processes This study has also identified enabling technologies which will need to be funded and developed for advanced human missions. These include development of high-efficiency electric propulsion systems, common bulk-head tanks, aluminum-air fuel cells, and advanced thermal protection system materials - - both ablative and reusable. 


\section{NOMENCLATURE}

Delta-IV-H = Delta-IV-Heavy expendable launch vehicle (a conceptual vehicle under consideration by Boeing)

ECLSS = Environmental Control Life Support System

EVA $=$ Extra Vehicular Activity

ISS = International Space Station

ISSRMS = International Space Station Remote Manipulator System

$\mathrm{JSC}=$ Johnson Space Center

$\mathrm{Km}=$ kilometer

$\mathrm{KW}=$ kilowatt

$\mathrm{L} 1=$ Lunar Libration point one

L1LHL $=$ L1 Lunar Habitat Lander

L1LL $=$ L1 Lunar Lander

LL1 $=$ Lunar Libration point one

$\dot{L} M=$ Apollo program Lunar Module

LOX $=$ Liquid oxygen

LTV $=$ Lunar Transfer Vehicle

$\mathrm{M}=$ meters

NASA $=$ National Aeronautics and Space Administration

PDGF $=$ Power and Data Grapple Fixture

Prox Ops $=$ Proximity operations

Psia $=$ Pounds per square inch absolute pressure

RCS $=$ Reaction Control System

RMS $=$ Remote Manipulator system

$\mathrm{Sec}=$ seconds

SEP $=$ Solar Electric Propulsion

TCS $=$ Thermal Control System

TEI $=$ Trans-earth Injection

TLI $=$ Trans-Iunar Injection

TPS $=$ Thermal Protection System

TRL $=$ Technology Readiness Level

\section{ACKNOWLEDGMENTS}

The author wishes to thank the JSC Advanced Design team for its dedication and support in the development of concepts leading to the designs of the spacecraft listed in this paper. It is through their efforts that conceptual designs for all the on-board vehicle subsystems were developed. The author would also like to thank the management of the NASA/Johnson Exploration Office for their support, oversight, and suggestions throughout the design process which spurred the team and the author to system engineering excellence.

\section{REFERENCES}

Braden, E., Low Thrust Study to the Cis-Lunar Libration Point, 2000

Fullerton, R., Overview of EVA Concepts For Large Deep Space Telescopes, 2001

Geffre, J., Lunar L1 Gateway Introduction Package, 2001

JSC Advanced Design Team, Gateway Habitat Configuration, 2001

JSC Advanced Design Team, L1 Lunar Lander Element Conceptual Design Report, 2000

JSC Advanced Design Team, L1 Lunar Habitat Lander Element Conceptual Design Report, 2000

JSC Advanced Design Team, Lunar Transfer Vehicle Element Conceptual Design Report, 2001

Tripathi, A., Assessment of the Current Earth's Neighborhood Architecture, 2001 